\title{
Rationalizability of menu preferences
}

\author{
Christopher J. Tyson 1
}

Received: 15 August 2016 / Accepted: 20 March 2017 / Published online: 31 March 2017

(C) The Author(s) 2017. This article is an open access publication, corrected publication February 2018

\begin{abstract}
The class of preferences over opportunity sets (“menus") rationalizable by underlying preferences over the alternatives is characterized for the general case in which the dataset is unrestricted. In particular, both the universal set of alternatives and the domain of menus over which preferences are asserted by the decision maker are arbitrary. The key "Cover Dominance" axiom states that any menu strictly preferred to a collection of menus must be strictly preferred to any menu covered by the collection. The method of characterization relies upon transitivity of menu preferences, but completeness can be relaxed.
\end{abstract}

Keywords General domains · Opportunity sets · Revealed preference · Transitivity

JEL Classification D01 - D11

\section{Introduction}

\subsection{The rationalizability question}

This paper studies the question of when observed preferences over opportunity sets ("menus") can be rationalized by underlying preferences over the alternatives they contain ("meals"). In the simplest environment, with finitely many options and a weak preference asserted by the decision maker between each pair of subsets of the universal

The original version of this article was revised: Author correction was misinterpreted and both the symbols (binary relations and negations) have been changed to negation symbols. Now, they have been corrected.

$凶$ Christopher J. Tyson

c.j.tyson@qmul.ac.uk

1 School of Economics and Finance, Queen Mary University of London, London E1 4NS, UK 
menu, conditions for rationalizability were given by Kreps (1979, pp. 565-566) as a benchmark for his axiomatization of "preference for flexibility." Yet despite a large subsequent literature that incorporates into menu preferences various other tastes and influences on behavior, the rationalizability question remains unanswered in the general case. ${ }^{1}$

We shall generalize the environment in Kreps (1979) along both dimensions mentioned in the previous paragraph. Firstly, any nonempty universal set of alternatives will be permitted, whether finite or (countably or uncountably) infinite. This will allow our framework to accommodate the many economic contexts in which finiteness is not a natural assumption, such as choice among consumption bundles, production plans, lotteries, and asset allocations. ${ }^{2}$ Secondly, the domain of menus over which preferences are asserted will be permitted to be any nonempty subset of the set of conceivable choice problems. This will make our findings applicable to arbitrary datasets, including those that arise from observational data or laboratory experiments; or from structured settings where all menus are, for example, budget or production sets.

At the heart of the rationalizability question is the need to translate meal preferences into menu preferences and vice versa. On the one hand, any meal-preference relation induces a menu-preference relation via a simple rule (see Definition 1): One menu is weakly preferred to another if each meal on the second menu is weakly inferior to some meal on the first menu. This relationship-which formalizes the concept of a rationalization-expresses the intuition that a menu is as good as the best meal it contains.

Moving in the opposite direction is trivial in full-domain environments, where meal preferences coincide with the observed preferences over singleton menus. But in our general framework, singleton menus need not be included in the domain, and therefore a more reliable notion of revealed meal preference is required. We propose the following conception (see Definition 3), which in a sense reverses the rule in the previous paragraph: One meal is weakly preferred to another if each menu containing the first meal is weakly superior to some menu containing the second meal. This revealed relation captures the intuition that a meal is as bad as the worst menu containing it, and will be used to replicate the observed menu preferences in proving our results.

\footnotetext{
${ }^{1}$ Barbera et al. (2004) survey the menu-preference literature, while models of temptation, in particular, are surveyed by Lipman and Pesendorfer (2013). Among the numerous more recent papers are those of Ahn and Sarver (2013), Dekel et al. (2009), Epstein et al. (2007), Olszewski (2007), and Stovall (2010). As mentioned by Dekel et al. (2009, p. 938), “[a] menu can be interpreted either literally or as an action which affects subsequent opportunities."

2 Note that we deliberately avoid "modeling the set of alternatives as lotteries and utilizing the resulting linear structure by imposing the von Neumann-Morgenstern axioms" (Gul and Pesendorfer 2001, p. 1406); a practice pioneered by Dekel et al. (2001) and Gul and Pesendorfer (2001) and adopted in much of the ensuing menu-preference literature. (Some exceptions include Ergin 2003, Gul and Pesendorfer 2005, and Nehring 1999). While it has the advantage of facilitating precise identification of model components, such as the subjective state space in Dekel et al. (2001), the lottery formulation can be viewed as a purely technical device to the extent that objective risk is not essential to the phenomenon of interest (e.g., temptation). Moreover, this formulation requires more of the decision maker, who must rank menus of lotteries over outcomes rather than simply menus of outcomes.
} 


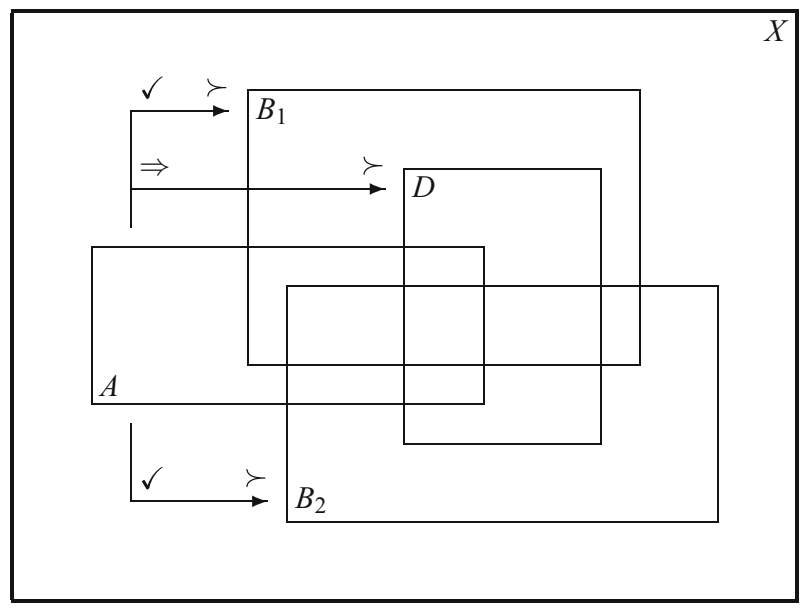

Fig. 1 The Cover Dominance axiom. The menus $A, B_{1}, B_{2}, D \subset X$ are in the domain of the menupreference relation $\succsim$, with $D \subset B_{1} \cup B_{2}$. If $A \succ B_{1}$ and $A \succ B_{2}$, then Cover Dominance requires that $A \succ D$

\subsection{Axioms for rationalizability}

In the finite, full-domain environment, Kreps (1979) characterizes the class of menu preferences rationalizable by complete and transitive meal preferences. The first of his two axioms, which we label Menu Order, states simply that the observed menu preferences are themselves complete and transitive. This is a straightforward consequence of the ordering properties imposed on the rationalizing meal-preference relation, and the argument does not depend on finiteness or the full-domain assumption (see Corollary 1). Hence, we inherit Menu Order from Kreps as a necessary condition.

The second axiom used by Kreps (1979, p. 566), which we label Kreps Consistency, states that the more preferred of any two menus is indifferent to their union. This condition is clearly unsuitable for our framework, in which the domain of the menu-preference relation need not be closed under union. We therefore replace Kreps Consistency with a new and somewhat stronger axiom, Cover Dominance, which is appropriate for the general case. ${ }^{3}$

To understand the content of Cover Dominance, consider the situation in Fig. 1. Here $\succsim$ is the weak menu-preference relation (with associated strict relation $\succ$ and indifference relation $\sim), X$ is the universal set of alternatives, and the menus $A, B_{1}, B_{2}, D \subset X$ are in the domain of $\succsim$. If both $A \succ B_{1}$ and $A \succ B_{2}$ and if $\succsim$ is rationalizable by meal preferences, then each meal in $B_{1} \cup B_{2}$ should be strictly inferior to a meal in $A$. If also $D \subset B_{1} \cup B_{2}$, then each meal in $D$ (the "covered" menu) should likewise

\footnotetext{
3 To be precise, Cover Dominance is logically stronger than Kreps Consistency in the presence of Menu Order (see Fig. 3).
} 
be strictly inferior to a meal in $A$. This leads us to anticipate that $A \succ D$, which is the conclusion mandated by Cover Dominance. ${ }^{4}$

Our main result (Theorem 1) thus characterizes rationalizability by means of the Menu Order and Cover Dominance conditions. In proving sufficiency of this axiom system, we also establish two of its implications that are of interest in their own right. One condition, Implicit Optima, states that each menu contains an alternative whose presence on any other menu guarantees that the second menu is no worse than the first. In terms of the rationalization, this means that even on infinite menus there can be found a greatest option with respect to the meal-preference relation. The second implied condition, Weak Cover Dominance, replaces strict with weak preference in both the hypotheses and the conclusion of Cover Dominance. This alternate version of the cover dominance property plays a role in the proof of sufficiency, as well as in linking our axiom system to that of Kreps (1979).

\subsection{Preferences over budget sets: an example}

For a concrete illustration of our framework and characterization result, let $X=\mathfrak{R}_{+}^{2}$ and imagine a consumer with endowment $\langle 1,1\rangle$ who may face a variety of different relative prices. Imagine further that the consumer asserts preferences over the four price vectors $\langle 1,1\rangle,\langle 1,2\rangle,\langle 2,1\rangle$, and $\langle 1,4\rangle$; with respective budget sets $B_{1}, B_{1 / 2}, B_{2}$, and $B_{1 / 4}$. This situation is depicted in Fig. 2, where the points $x^{1}, x^{2}, \ldots, x^{7}$ represent arbitrary consumption bundles in different regions of $X$.

Suppose first that $B_{1 / 2} \succ B_{2} \succ B_{1} \succ B_{1 / 4}$. Since $B_{1 / 2} \subset B_{1} \cup B_{1 / 4}$ and both $B_{2} \succ B_{1}$ and $B_{2} \succ B_{1 / 4}$, Cover Dominance requires that $B_{2} \succ B_{1 / 2}$. But this contradicts the observed preference $B_{1 / 2} \succ B_{2}$, so Cover Dominance fails in this case and we can conclude that $\succsim$ is not rationalizable by complete and transitive meal preferences.

Now, suppose instead that $B_{1 / 4} \succ B_{2} \succ B_{1} \succ B_{1 / 2}$. Since $B_{1} \subset B_{2} \cup B_{1 / 2}$ and both $B_{1 / 4} \succ B_{2}$ and $B_{1 / 4} \succ B_{1 / 2}$, Cover Dominance implies $B_{1 / 4} \succ B_{1}$. This agrees with the observed preferences, and it is straightforward to verify that no other violations of Cover Dominance can be found in the dataset. Of course Menu Order also holds, and thus $\succsim$ can be rationalized in this case.

Note that, according to the preferences in the previous paragraph, each of the menus containing $x^{5}$ (namely, $B_{1}$ and $B_{2}$ ) is strictly superior to some menu containing $x^{4}$ (namely, $B_{1 / 2}$ ). Evaluating each meal by the worst menu containing it, our revealed preference relation therefore considers $x^{5}$ strictly superior to $x^{4}$. Similarly, our relation considers $x^{7}$ strictly superior to $x^{5} ; x^{6}$ strictly superior to $x^{7}$; and $x^{1}, x^{2}, x^{3}$, and $x^{4}$ indifferent to each other since they are all members of the lowest ranked menu $\left(B_{1 / 2}\right)$.

The meal preferences that rationalize a given menu-preference relation will not in general be unique. ${ }^{5}$ Indeed, writing a generic consumption bundle in the present

\footnotetext{
4 Observe that menu $A$ is never compared directly to $B_{1} \cup B_{2}$, which need not be in the domain of $\succsim$. Moreover, note that Cover Dominance allows arbitrary (not only binary) unions of covering menus.

5 This is a natural consequence of our objective of characterizing rationalizability over arbitrary domains, as evidenced by the similar non-uniqueness seen in Richter (1966), Bossert et al. (2006), Tyson (2013), and other contributions that share this goal.
} 


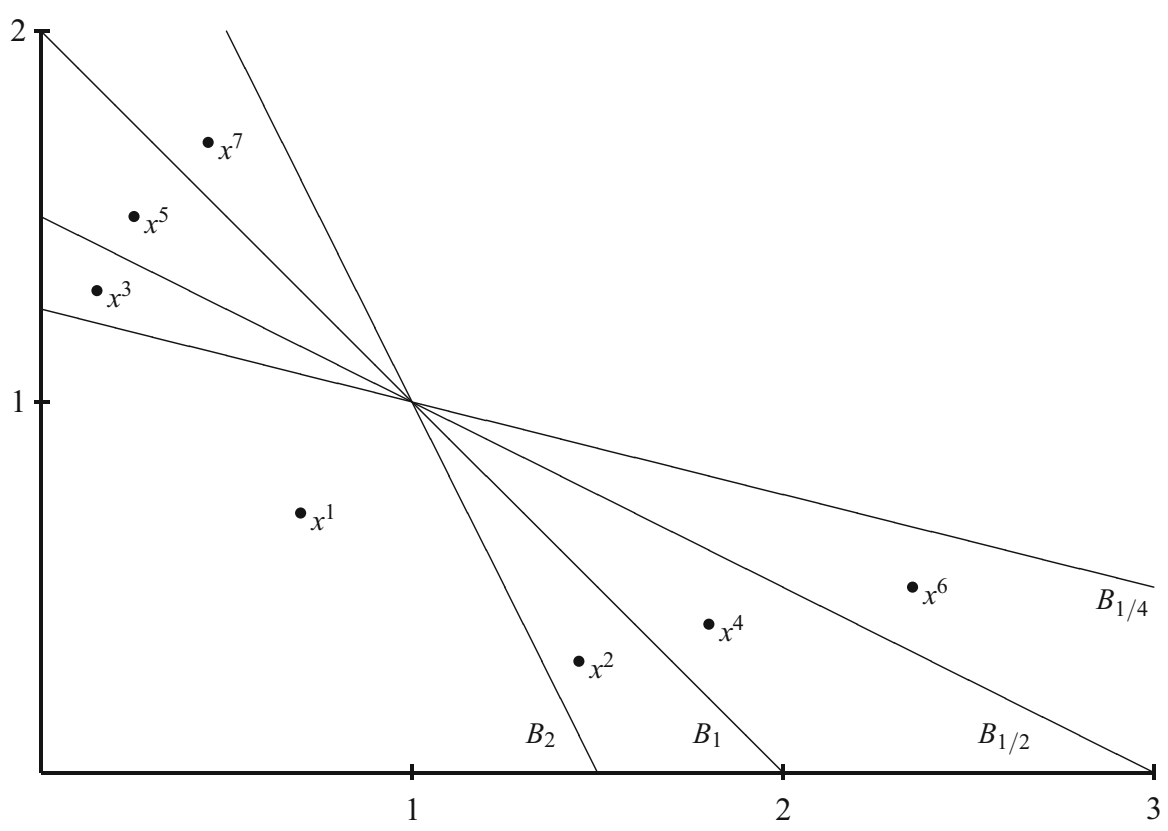

Fig. 2 An example of preferences over the budget sets $B_{1}, B_{1 / 2}, B_{2}$, and $B_{1 / 4}$. The order $B_{1 / 2} \succ B_{2} \succ$ $B_{1} \succ B_{1 / 4}$ violates Cover Dominance and thus is not rationalizable. In contrast, the order $B_{1 / 4} \succ B_{2} \succ$ $B_{1} \succ B_{1 / 2}$ satisfies Cover Dominance and thus is rationalizable

context as $z=\left\langle z_{1}, z_{2}\right\rangle$, the preferences $B_{1 / 4} \succ B_{2} \succ B_{1} \succ B_{1 / 2}$ can be rationalized by the two distinct meal-preference relations represented by the utility functions $u(z)=$ $19 z_{1}+30 z_{2}$ and $v(z)=\max \left\{10 z_{1} z_{2}^{3}, z_{1}^{4} z_{2}\right\}$. Neither coincides with our revealed mealpreference relation, which in this case has just four indifference classes (corresponding to the four menus in the dataset). Concretely, the alternatives $\tilde{x}^{1}=\langle 0.2,1.0\rangle$ and $\tilde{x}^{4}=\langle 2.0,0.1\rangle$ are ranked as indifferent by our revealed relation, while the two utility functions yield opposing strict preferences computed as $u\left(\tilde{x}^{1}\right)=33.8<41.0=$ $u\left(\tilde{x}^{4}\right)$ and $v\left(\tilde{x}^{1}\right)=2.0>1.6=v\left(\tilde{x}^{4}\right)$. This example makes clear that deducing the decision maker's true meal preferences from arbitrary menu-preference data will not typically be possible; and doing so is not our purpose in this paper. We wish, rather, to find concise and transparent conditions that characterize rationalizability without the help of domain assumptions and thus allow us to test this hypothesis under more realistic circumstances.

\subsection{Road map}

The remainder of the paper is structured as follows. Section 2 describes how menu preferences are induced by meal preferences and how meal preferences are revealed by menu preferences, and introduces the Menu Order axiom. Section 3 proceeds to develop the Cover Dominance axiom and to state and outline the proof of our main result. Section 4 discusses rationalizability by incomplete meal preferences, shows 
how Kreps's original characterization can be derived as a corollary of our result, and demonstrates how the theory of rationalizable menu preferences parallels the theory of rationalizable choice functions. Proofs are in the "Appendix".

\section{Meal and menu preferences}

\subsection{Preliminaries}

Let $X$ be a nonempty set of alternatives (also called "options" or "meals"), write $\mathfrak{X}$ for the power set of $X$, and fix both a nonempty domain $\mathfrak{D} \subset \mathfrak{X} \backslash\{\emptyset\}$ of menus and a relation $\succsim$ on $\mathfrak{D}$. Our primitives are thus $\langle X, \mathfrak{D}, \succsim\rangle$. Write $\mathfrak{D}_{x}=\{A \in \mathfrak{D}: x \in A\}$ for the set of menus that contain option $x$.

Given a relation $\mathrm{R}$ on $X$, write $G(A, \mathrm{R})=\{x \in A: \forall y \in A \quad x \mathrm{R} y\}$ for the set of $\mathrm{R}$-greatest alternatives on menu $A$. A relation is a preorder if it is both reflexive and transitive. For brevity, a complete preorder will be referred to simply as an order. ${ }^{6}$

As usual, we write $A \sim B$ when $A \succsim B \succsim A$ and $A \succ B$ when $A \succsim B \succsim A$. Likewise, we write $x \mathrm{I} y$ when $x \mathrm{R} y \mathrm{R} x$ and $x \mathrm{P} y$ when both $x \mathrm{R} y$ and $\neg y \mathrm{R} x$.

\subsection{Induced menu preferences}

Kreps (1979, p. 565) uses preferences over alternatives to define preferences over menus "in the obvious fashion."

Definition 1 Given a relation $\mathrm{R}$ on $X$, define a relation $\succsim_{\mathrm{R}}$ on $\mathfrak{D}$ as follows: For each $A, B \in \mathfrak{D}$, let $A \succsim_{\mathrm{R}} B$ if and only if $\forall y \in B$, there exists an $x \in A$ such that $x \mathrm{R} y$.

In words, the induced relation $\succsim_{\mathrm{R}}$ weakly prefers menu $A$ to $B$ if each option on $B$ is weakly inferior, according to the meal-preference relation $\mathrm{R}$, to some option on $A$. This is consistent with the standard model of choice, in which the decision maker will eventually select from each menu a preference-maximal option according to which the menu itself may be valued.

We write $A \sim_{\mathrm{R}} B$ when $A \succsim_{\mathrm{R}} B \succsim_{\mathrm{R}} A$ and $A \succ_{\mathrm{R}} B$ when $A \succsim_{\mathrm{R}} B \succsim_{\mathrm{R}} A$. For complete R, Definition 1 can then be expressed as $B \succ_{\mathrm{R}} A$ if and only if $\exists y \in B$ such that $\forall x \in A$ we have $y \mathrm{P} x$. That is, a strict menu preference for $B$ over $A$ is induced by $\mathrm{R}$ if some option on $B$ is strictly better than every option on $A$.

An important consequence of Definition 1 is that the induced relation $\succsim_{R}$ inherits a number of ordering properties from $\mathrm{R}$.

Proposition 1 A. If $\mathrm{R}$ is reflexive, then $\succsim_{\mathrm{R}}$ is reflexive. $\mathrm{B}$. If $\mathrm{R}$ is complete, then $\succsim_{\mathrm{R}}$ is complete. $\mathbf{C}$. If $\mathrm{R}$ is transitive, then $\succsim_{\mathrm{R}}$ is transitive.

Finally, we can use induced menu preferences to formalize our concept of rationalizability.

\footnotetext{
${ }^{6}$ Recall that a binary relation $\mathrm{R}$ on $X$ is reflexive if $\forall x \in X$ we have $x \mathrm{R} x$; transitive if $\forall x, y, z \in X$ we have $x \mathrm{R} y \mathrm{R} z \Longrightarrow x \mathrm{R} z$; and complete if $\forall x, y \in X$ we have $\neg x \mathrm{R} y \Longrightarrow y \mathrm{R} x$.
} 
Definition 2 A rationalization of $\succsim$ is a relation $\mathrm{R}$ on $X$ such that $\succsim_{=} \succsim_{\mathrm{R}}$.

If the unobserved meal-preference relation is complete and transitive, then it follows from Proposition 1 that the induced menu-preference relation exhibits the same properties. This yields a necessary condition for rationalizability by an order in the general case.

Condition 1 (Menu Order) The relation $\succsim$ is an order.

Corollary 1 If $\succsim$ is rationalized by an order, then Menu Order holds.

Example 1 Let $X=w x y z$ and $\mathfrak{D}=\{z, w x, w z, x y, x z, y z, x y z\}$. Then, the order $w \mathrm{P} x \mathrm{I} y \mathrm{P} z$ on meals induces the order $w x \sim_{\mathrm{R}} w z \succ_{\mathrm{R}} x y \sim_{\mathrm{R}} x z \sim_{\mathrm{R}} y z \sim_{\mathrm{R}} x y z \succ_{\mathrm{R}} z$ on menus. For instance, we have that $w z \succsim_{\mathrm{R}} x y$ since $w \mathrm{R} x$ and $w \mathrm{R} y$, while $x y \succsim_{\mathrm{R}} w z$ since $\neg x \mathrm{R} w$ and $\neg y \mathrm{R} w .^{7}$

\subsection{Revealed meal preferences}

In order to achieve the desired characterization, we will also need to be able to translate the decision maker's tastes from the menu-preference relation $\succsim$ to a revealed mealpreference relation. This is accomplished by the following construction.

Definition 3 Define a relation $\hat{\mathrm{R}}$ on $X$ as follows: For each $x, y \in X$, let $x \hat{\mathrm{R}} y$ if and only if $\forall A \in \mathfrak{D}_{x}$, there exists a $B \in \mathfrak{D}_{y}$ such that $A \succsim B$.

Here, the revealed relation $\hat{\mathrm{R}}$ weakly prefers option $x$ to $y$ if each menu containing $x$ is no worse, according to the primitive relation $\succsim$, than some menu containing $y$.

We write $x \hat{\mathrm{I}} y$ when $x \hat{\mathrm{R}} y \hat{\mathrm{R}} x$ and $x \hat{\mathrm{P}} y$ when both $x \hat{\mathrm{R}} y$ and $\neg y \hat{\mathrm{R}} x$. For complete $\succsim$, Definition 3 can then be expressed as $y \hat{\mathrm{P}} x$ if and only if $\exists A \in \mathfrak{D}_{x}$ such that $\forall B \in \widetilde{\mathfrak{D}}_{y}$ we have $B \succ A$. That is, a strict meal preference for $y$ over $x$ is revealed by $\succsim$ if some menu containing $x$ is strictly worse than every menu containing $y$.

The latter paraphrasing of Definition 3 conveys the rationale behind the revealed meal-preference relation $\hat{\mathrm{R}}$ : If even the worst menu $B$ containing $y$ is strictly preferred to some menu $A$ containing $x$, this suggests that $y$ itself is strictly better than everything in $A$ and in particular strictly better than $x$. The expression $x \hat{\mathrm{R}} y$ records the absence of this situation, where the evidence from $\succsim$ indicates instead that $x$ is at least as good as $y$.

Our next result is the meal-preference analog of Proposition 1, establishing that $\hat{R}$ inherits the same ordering properties from $\succsim$.

Proposition 2 A. If $\succsim$ is reflexive, then $\hat{\mathrm{R}}$ is reflexive. B. If $\succsim$ is complete, then $\hat{\mathrm{R}}$ is complete. C. If $\succsim$ is transitive, then $\hat{\mathrm{R}}$ is transitive.

Corollary 2 Menu Order implies that $\hat{\mathrm{R}}$ is an order.

\footnotetext{
7 Note in this example the multiplicative notation for enumerated sets, which we use when convenient.
} 
Example 2 For the domain defined in Example 1, we have $\mathfrak{D}_{w}=\{w x, w z\}, \mathfrak{D}_{x}=$ $\{w x, x y, x z, x y z\}, \mathfrak{D}_{y}=\{x y, y z, x y z\}$, and $\mathfrak{D}_{z}=\{z, w z, x z, y z, x y z\}$. In this case, the order $w x \sim w z \succ x y \sim x z \sim y z \sim x y z \succ z$ on menus (identical to the induced preferences in Example 1) reveals the original order $w \hat{\mathrm{P}} x \hat{\mathrm{I}} y \hat{\mathrm{P}} z$ on meals. For instance, we have that $w \hat{\mathrm{R}} x$ since $w x \succsim x y$ and $w z \succsim x y$, while $\neg x \hat{\mathrm{R}} w$ since $x y \succsim w x$ and $x y \succsim w z$.

\section{Main result}

\subsection{Characterization of rationalizability}

We know from Corollary 2 that Menu Order is sufficient for $\hat{R}$ to be complete and transitive. Hence, what is needed is a further condition that together with Menu Order will guarantee that this relation rationalizes the observed $\succsim$.

To construct the required axiom, we shall use the concept of a covering set of menus.

Definition 4 The set $\mathfrak{B} \subset \mathfrak{D}$ is said to cover $A \in \mathfrak{D}$ if $A \subset \bigcup \mathfrak{B}:=\bigcup_{B \in \mathfrak{B}} B$.

Our condition then states that any menu strictly preferred to the elements of a cover must be strictly preferred to the target of the cover.

Condition 2 (Cover Dominance) Let $A, D \in \mathfrak{D}$ and let $\mathfrak{B} \subset \mathfrak{D}$ cover $D$. If for each $B \in \mathfrak{B}$ we have $A \succ B$, then $A \succ D$.

Here, the intuition is that $\mathfrak{B}$ collectively should be no worse than $D$, so Cover Dominance has the flavor of a transitivity condition. Note, however, that $\bigcup \mathfrak{B}$ may or may not be in $\mathfrak{D}$, so we cannot argue simply that $A \succ \bigcup \mathfrak{B} \succsim D$ and hence $A \succ D$.

To show necessity of our new axiom, we shall need the set of R-greatest elements of each menu to be nonempty. Implicitly, this is of course the set of eventual choices from the menu, and hence the additional structure required amounts to an assumption of nonempty-valued choice.

Proposition 3 If $\succsim$ is rationalized by an order $\mathrm{R}$ with $G(\cdot, \mathrm{R})$ nonempty, then Cover Dominance holds.

Our main result combines the assumptions on meal preference and the conditions on menu preference across Corollary 1 and Proposition 3.

Theorem 1 The relation $\succsim$ is rationalized by an order $\mathrm{R}$ with $G(\cdot, \mathrm{R})$ nonempty if and only if Menu Order and Cover Dominance hold.

Example 3 The menu preferences in Example 2 are rationalized by an order and hence satisfy both Menu Order and Cover Dominance. For instance, we have $x z \subset x y z=$ $x y \cup y z, w z \succ x y$, and $w z \succ y z$, so Cover Dominance requires that $w z \succ x z$ (which is in fact the case). In contrast, the preferences $w x \sim w z \sim x z \succ x y \sim y z \sim x y z \succ z$ fail Cover Dominance and so cannot be rationalized by an order. ${ }^{8}$

\footnotetext{
8 To see this, note that for any order rationalization $\mathrm{R}$ we have that $w x>x y \Longrightarrow w \mathrm{P} x$ and $w z \succ z \Longrightarrow$ $w \mathrm{P} z$. But this would imply that $w x \succ x z$ (which is in fact not the case).
} 


\subsection{Sufficiency of axioms}

To achieve our characterization, it remains to show that the axioms in Theorem 1 are sufficient for $\hat{\mathrm{R}}$ to rationalize $\succsim$ and generate a nonempty $G(\cdot, \hat{\mathrm{R}})$. The former property means both that all observed preferences are faithfully reproduced by $\hat{\mathrm{R}}$, written $\succsim \subset \succsim_{\hat{R}}$; and that all preferences induced by $\hat{R}$ are genuine, written $\succsim_{\hat{R}} \subset \succsim^{9}$.

We shall verify the required properties of $\hat{R}$ with the help of two auxiliary conditions implied by our axiom system. The first asserts the existence within each menu of an "implicit optimum" whose appearance on any other menu ensures weak menusuperiority. ${ }^{10}$

Condition 3 (Implicit Optima) For each $A \in \mathfrak{D}$, there exists an $x \in A$ such that $\forall B \in \mathfrak{D}_{x}$ we have $B \succsim A$.

Proposition 4 A. If $\succsim$ is complete, then Cover Dominance implies Implicit Optima. B. Menu Order and Implicit Optima imply Cover Dominance.

This condition yields the desired nonemptiness property of $\hat{R}$.

Proposition 5 Implicit Optima implies that $G(\cdot, \hat{\mathrm{R}})$ is nonempty.

Conveniently, it can also be used to prove the faithful-reproduction property.

Proposition 6 If $\succsim$ is transitive, then Implicit Optima implies that $\succsim \subset \succsim_{\hat{\mathrm{R}}}$.

Example 4 Recall the menu-preference order $\succsim$ defined in Example 3, for which Cover Dominance fails. Here, alternative $w$ is an implicit optimum for the menus $w x$ and $w z$, alternative $x$ for the menus $x y$ and $x y z$, alternative $y$ for the menu $y z$, and alternative $z$ for the menu $z$. The menu $x z$ contains no implicit optimum, since $x \in x y \prec x z$ and $z \in y z \prec x z$.

Our second auxiliary condition is a weak-preference counterpart of Cover Dominance and has a similar intuition in terms of the cover $\mathfrak{B}$ supplying a bridge between menu $A$ and the (now weakly) inferior menu $D$.

Condition 4 (Weak Cover Dominance) Let $A, D \in \mathfrak{D}$ and let $\mathfrak{B} \subset \mathfrak{D}$ cover $D$. If for each $B \in \mathfrak{B}$ we have $A \succsim B$, then $A \succsim D$.

Proposition 7 If $\succsim$ is transitive, then Implicit Optima implies Weak Cover Dominance.

\footnotetext{
9 This is different from the statement that $\hat{R}$ coincides with the decision maker's true but unobserved meal-preference relation R. Even when $\succsim_{\hat{R}}=\succsim=\succsim_{R}$ and we have successfully replicated the agent's menu preferences, we cannot be certain that either $R \subset \hat{R}$ or $\hat{R} \subset R$. Indeed, the failure of these inclusions in general is made clear by the example of preferences over budget sets in Sect. 1.3.

10 This condition strengthens the Desire for Commitment axiom used by Dekel et al. (2009, p. 946) to study "temptation-driven preferences." In the full-domain environment, Desire for Commitment requires that for each $A \in \mathfrak{D}$ there exists an $x \in A$ such that $\{x\} \succsim A$. Here, alternative $x$ can be interpreted as an implicit optimum for menu $A$, but since Dekel et al. allow for temptation they do not require $B \succsim A$ for menus $B \in \mathfrak{D}_{x}$ other than the singleton $\{x\}$.
} 
This condition can be used to prove the genuineness property of $\hat{\mathrm{R}}$.

Proposition 8 Weak Cover Dominance implies that $\succsim_{\hat{R}} \subset \succsim$.

Note that Weak Cover Dominance is not in general strong enough to yield Implicit Optima, even in the presence of Menu Order. To support this claim, we offer the following example.

Example 5 Let $X=x_{1} y_{1} x_{2} y_{2} x_{3} y_{3} \ldots, A=x_{1} x_{2} x_{3} \ldots, B_{k}=x_{k} y_{k}$ for $k \geq 1$, and $\mathfrak{D}=\left\{A, B_{1}, B_{2}, B_{3}, \ldots\right\}$. Moreover, let $B_{1} \prec B_{2} \prec B_{3} \prec \ldots$, and let $A \succ B_{k}$ for $k \geq 1$. While these preferences satisfy both Menu Order and Weak Cover Dominance, they fail Implicit Optima. Indeed, the menu $A$ contains no implicit optimum since $x_{k} \in B_{k} \prec A$ for $k \geq 1$. Note that, in view of Proposition $4 \mathrm{~A}, \succsim$ must fail Cover Dominance as well. This can be verified by observing that $A \subset \bigcup_{k=1}^{\infty} B_{k}$ and $A \succ B_{k}$ for $k \geq 1$, while the conclusion $A \succ A$ is obviously false.

To show that $\hat{\mathrm{R}}$ rationalizes $\succsim$, we need the full strength of Implicit Optima and not just Weak Cover Dominance (see Propositions 6-8). Under Menu Order, we know that Implicit Optima and Cover Dominance are equivalent (see Proposition 4), but to ensure these conditions hold rationalizability alone is insufficient-nonemptiness is also needed (see Proposition 3 ). Fortunately, nonemptiness of $G(\cdot, \hat{\mathrm{R}})$ is guaranteed by Implicit Optima (see Proposition 5), making possible the construction of a two-way result in Theorem $1 .^{11}$

\section{Additional results and discussion}

\subsection{Incomplete preferences}

Theorem 1 can be adapted relatively easily to accommodate incompleteness of the primitive relation $\succsim$ and the rationalizing relation $\mathrm{R}$. Propositions $1-2$ show that the properties of a preorder (namely, reflexivity and transitivity) transfer between meal and menu preferences independently of completeness, and hence the following condition is a suitable adaptation.

Condition 5 (Menu Preorder) The relation $\succsim$ is a preorder.

Corollary 3 A. If $\succsim$ is rationalized by a preorder, then Menu Preorder holds. B. Menu Preorder implies that $\hat{\mathrm{R}}$ is a preorder.

As for Cover Dominance, scrutiny of the proof of Theorem 1 reveals that a slightly different version of the axiom yields a valid characterization with or without the completeness assumption. ${ }^{12}$

\footnotetext{
11 Example 5 illustrates why Theorem 1 imposes the nonemptiness condition. At a somewhat deeper level, this condition is needed because our theory of rationalizable menu preferences parallels the theory of rationalizable choice functions (see Sect. 4.3). In the latter context, nonemptiness of each set of maximal alternatives is typically imposed as a background assumption, whereas we state the property explicitly.

12 On the other hand, there is little prospect of relaxing transitivity, which is used heavily in the proof of Theorem 1. For instance, transitivity is employed to establish the necessity of Cover Dominance (in Proposition 3 ) and to show the faithful-reproduction property of $\hat{\mathrm{R}}$ (in Proposition 6).
} 
Condition 6 (Negative Cover Dominance) Let $A, D \in \mathfrak{D}$ and let $\mathfrak{B} \subset \mathfrak{D}$ cover $D$. If for each $B \in \mathfrak{B}$ we have $B \succsim A$, then $D \succsim A$.

When $\succsim$ is complete, we have both $B \succsim A \Longleftrightarrow[A \succsim B \wedge B \succsim A] \Longleftrightarrow A \succ B$ and $D \succsim A \Longleftrightarrow[A \succsim D \wedge D \succsim A] \Longleftrightarrow A \succ D$; and so the two versions of the condition are logically equivalent. In this case, we favor Cover Dominance since it is the more transparent and readily interpretable form of the axiom, but adapting our result to the incomplete case calls for the alternative form.

Theorem 2 The relation $\succsim$ is rationalized by a preorder $\mathrm{R}$ with $G(\cdot, \mathrm{R})$ nonempty if and only if Menu Preorder and Negative Cover Dominance hold.

The proof of this result requires only minor changes to that of Theorem 1 and is therefore left to the reader.

\subsection{Domain restrictions}

Our main result characterizes rationalizability of menu preferences using the Menu Order and Cover Dominance axioms. To establish sufficiency, we have shown that in the presence of Menu Order, Cover Dominance is equivalent to Implicit Optima, which in turn implies Weak Cover Dominance. But Weak Cover Dominance is not in general strong enough to yield Implicit Optima, even with Menu Order. For this to be the case, we need to assume that the domain $\mathfrak{D}$ is finite, as established by the following proposition.

Proposition 9 Let $\mathfrak{D}$ be finite. Then, Menu Order and Weak Cover Dominance imply Implicit Optima.

Assume now that the domain $\mathfrak{D}$ is both finite and closed under union. ${ }^{13}$ This takes us into a setting where rationalizability is captured by the axiom originally proposed by Kreps (1979, p. 565).

Condition 7 (Kreps Consistency) Let $A, B \in \mathfrak{D}$ be such that $A \cup B \in \mathfrak{D}$. If $A \succsim B$, then $A \sim A \cup B$.

Proposition 10 If $\succsim$ is reflexive, then Weak Cover Dominance implies Kreps Consistency.

A straightforward implication of Kreps's condition is monotonicity with respect to set inclusion (called "desire for flexibility" in Kreps 1979).

Condition 8 (Monotonicity) Let $A, B \in \mathfrak{D}$. If $A \subset B$, then $B \succsim A$.

Proposition 11 If $\succsim$ is complete, then Kreps Consistency implies Monotonicity.

13 A referee points out that when $\mathfrak{D}$ is finite, the assumption that it is closed under union is substantially less restrictive. This is because a rationalization $\mathrm{R}$ of menu preferences over any finite $\mathfrak{D}$ can be extended to the closure of $\mathfrak{D}$ under union, and moreover, nonemptiness of $G(\cdot, \mathrm{R})$ will survive this extension. The same is not true for infinite $\mathfrak{D}$ (cf. Example 5). 


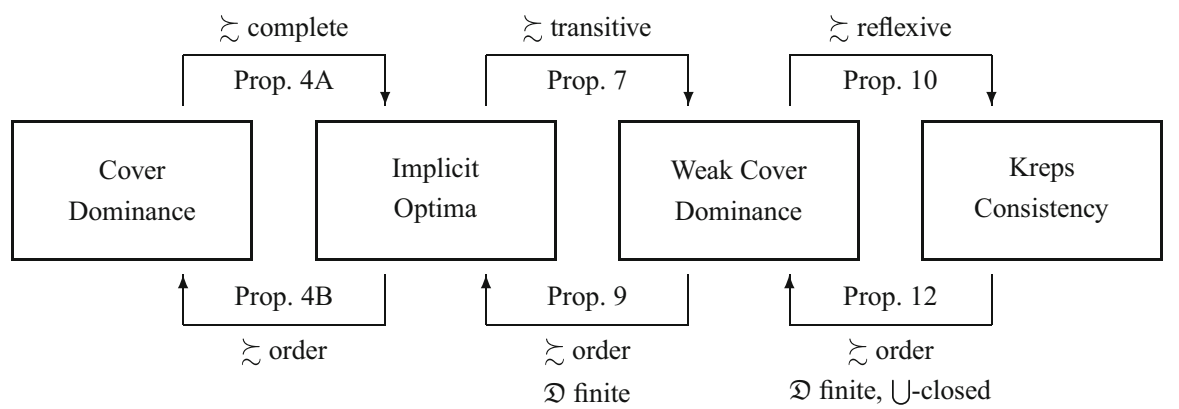

Fig. 3 Logical relationships between selected axioms. Each implication is labeled with the relevant result

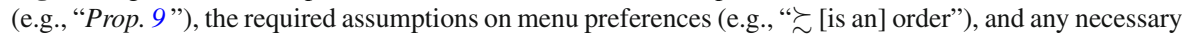
restrictions on the domain (e.g., " $\mathfrak{D}[$ is] finite")

The latter fact is useful in proving the following converse to Proposition 10.

Proposition 12 Let $\mathfrak{D}$ be finite and closed under union. Then, Menu Order and Kreps Consistency imply Weak Cover Dominance.

We can then state a version of our result that has Kreps's full-domain characterization as an immediate corollary. ${ }^{14}$

Theorem 3 Let $\mathfrak{D}$ be finite and closed under union. Then, $\succsim$ is rationalized by an order $\mathrm{R}$ with $G(\cdot, \mathrm{R})$ nonempty if and only if Menu Order and Kreps Consistency hold.

Corollary 4 (Kreps 1979) Let $X$ be finite and $\mathfrak{D}=\mathfrak{X} \backslash\{\emptyset\}$. Then, $\succsim$ is rationalized by an order if and only if Menu Order and Kreps Consistency hold.

Selected axioms and implications are summarized in Fig. 3. When the domain is both finite and closed under union, any of the four conditions shown suffices (together with Menu Order) to characterize rationalizability. If the domain is not closed under union, then Kreps Consistency no longer suffices, and if $\mathfrak{D}$ is not finite then Weak Cover Dominance too is inadequate. For general domains, the desired axiomatization is supplied by either Cover Dominance or Implicit Optima. The latter condition employs an existential quantifier and so can be seen as less attractive in terms of falsifiability. For this reason, we use Cover Dominance in the statement of Theorem $1 .{ }^{15}$

\subsection{Analogy with rationalizability of choice functions}

Several aspects of our investigation of rationalizable menu preferences have counterparts in the theory of rationalizable choice functions. Here, we briefly outline the

\footnotetext{
14 Theorem 3 follows from Theorem 1 together with Propositions 4, 7, 9, 10, and 12.

15 For a penetrating analysis of the structure of axioms and falsifiability of the associated theories, see Chambers et al. (2014).
} 
analogy between these two frameworks, assuming in the text for expository purposes that the domain $\mathfrak{D}$ is both finite and closed under union.

A choice function over $\mathfrak{D}$ is a $C: \mathfrak{D} \rightarrow \mathfrak{X} \backslash\{\emptyset\}$ such that $\forall A \in \mathfrak{D}$ we have $C(A) \subset A$. The members of $C(A)$ are interpreted as the options chosen from menu $A$. A rationalization of $C$ is a relation $\mathrm{R}$ on $X$ such that $C=G(\cdot, \mathrm{R})$. Define the revealed meal-preference relation $\overline{\mathrm{R}}$ by $x \overline{\mathrm{R}} y$ if and only if $\exists A \in \mathfrak{D}_{y}$ such that $x \in C(A)$.

Recall that Weak Cover Dominance requires any menu weakly preferred to the elements of a cover to be weakly preferred to the target of the cover. The choice-theoretic counterpart of this requirement is a condition referred to by Tyson (2013, p. 955) as "extraction consistency": Any alternative chosen from the elements of a cover must be chosen (if available) from the target of the cover. ${ }^{16}$ Extraction consistency is necessary and sufficient for $C$ to admit a rationalization, just as Weak Cover Dominance is necessary and sufficient for an order $\succsim$ to admit a rationalization. Indeed, extraction consistency holds if and only if $C=G(\cdot, \overline{\mathrm{R}})$, just as Weak Cover Dominance holds if and only if $\succsim=\succsim_{\hat{R}}$.

Extraction consistency is equivalent to the conjunction of two conditions: The first, "contraction consistency," says that any meal chosen from a larger menu must be chosen (if available) from a smaller menu, and is the analog of Monotonicity. The second, "weak expansion consistency," says that any meal chosen from each menu in a collection must be chosen from the union of the collection, and is the analog of the following menu-preference axiom. ${ }^{17}$

Condition 9 (Weak Union Dominance) Let $A \in \mathfrak{D}$ and let $\mathfrak{B} \subset \mathfrak{D}$ be such that $\bigcup \mathfrak{B} \in \mathfrak{D}$. If for each $B \in \mathfrak{B}$ we have $A \succsim B$, then $A \succsim \bigcup \mathfrak{B}$.

We can now state a counterpart to the equivalence result for choice functions.

Proposition 13 If $\mathfrak{D}$ is closed under union and $\succsim$ is a preorder, then Weak Cover Dominance is equivalent to the conjunction of Monotonicity and Weak Union Dominance.

With regard to rationalizability, there are two notable differences between the menupreference and choice-function frameworks. The first concerns the ordering properties of the rationalizing relation $\mathrm{R}$. Transitivity of $\mathrm{R}$ is needed for our main result, in contrast to the characterization of rationalizable choice functions via extraction consistency. Moreover, in view of Propositions 1-2 we can ensure that $\mathrm{R}$ has the relevant ordering properties simply by imposing these same properties on $\succsim$, without modifying Cover Dominance. This differs from the choice-function setting, where extraction consistency must be strengthened to guarantee the existence of an order rationalization. ${ }^{18}$

\footnotetext{
16 Formally, for each $D \in \mathfrak{D}$ and for any cover $\mathfrak{B} \subset \mathfrak{D}$ of $D$, we have $\left[\bigcap_{B \in \mathfrak{B}} C(B)\right] \cap D \subset C(D)$. This is equivalent to the "V-Axiom" in Richter (1971, p. 33), apparently the first statement of the condition.

17 Formally, contraction consistency requires that for each $A, B \in \mathfrak{D}$ with $A \subset B$ we have $C(B) \cap$ $A \subset C(A)$, while weak expansion consistency requires that for each $\mathfrak{B} \subset \mathfrak{D}$ with $\bigcup \mathfrak{B} \in \mathfrak{D}$ we have $\bigcap_{B \in \mathfrak{B}} C(B) \subset C(\cup \mathfrak{B})$. These conditions are, respectively, "Property $\alpha$ " in Sen $(1969$, p. 384) and "Property $\gamma$ " in Sen (1971, p. 314).

18 The Congruence Axiom in Richter (1966, p. 637) is the classical condition achieving this goal.
} 
The second difference concerns the existence of an R-greatest alternative on each menu. In the menu-preference setting, we deal with this issue directly, proving (in Proposition 4A) that the Implicit Optima condition follows from our axiomatization and including nonemptiness of $G(\cdot, \mathrm{R})$ in the statement of our results. Indeed, it is to capture precisely this requirement that we use Cover Dominance in Theorem 1 rather than Weak Cover Dominance (the more direct analog of extraction consistency). In the choice-function setting, on the other hand, nonemptiness of $G(\cdot, \mathrm{R})$ is ensured by nonemptiness of the primitive $C$ together with the definition of a rationalization, independently of any axioms imposed.

Despite these differences, the theories of rationalizability for menu preferences and for choice functions have a considerable amount in common when formulated to allow arbitrary datasets, and this analogy may prove fruitful for future work in both areas.

Acknowledgements The author would like to thank Andrew Ellis, Marco Mariotti, Sujoy Mukerji, and an anonymous referee for useful comments and suggestions.

Open Access This article is distributed under the terms of the Creative Commons Attribution 4.0 International License (http://creativecommons.org/licenses/by/4.0/), which permits unrestricted use, distribution, and reproduction in any medium, provided you give appropriate credit to the original author(s) and the source, provide a link to the Creative Commons license, and indicate if changes were made.

\section{Appendix: Proofs}

Proof of Proposition 1 A. For all $A \in \mathfrak{D}$ and $\forall x \in A$, we have $x \mathrm{R} x$, since $\mathrm{R}$ is reflexive, and hence $A \succsim_{\mathrm{R}} A$. Thus, $\succsim_{\mathrm{R}}$ is reflexive. B. For all $A, B \in \mathfrak{D}$, we have

$$
\begin{aligned}
A \succsim_{\mathrm{R}} B & \Longleftrightarrow \neg \forall y \in B \quad \exists x \in A \quad x \mathrm{R} y \\
& \Longleftrightarrow \exists y \in B \quad \forall x \in A \quad \neg x \mathrm{R} y \\
& \Longleftrightarrow \exists y \in B \quad \forall x \in A \quad y \mathrm{R} x \\
& \Longleftrightarrow \forall x \in A \quad \exists y \in B \quad y \mathrm{R} x \\
& \Longleftrightarrow B \succsim_{\mathrm{R}} A,
\end{aligned}
$$

where the third implication uses the completeness of R. Thus, $\succsim_{\mathrm{R}}$ is complete. C. For all $A, B, D \in \mathfrak{D}$, we have

$$
\begin{aligned}
& A \succsim_{\mathrm{R}} B \succsim_{\mathrm{R}} D \Longleftrightarrow[\forall y \in B \quad \exists x \in A \quad x \mathrm{R} y] \wedge\left[\begin{array}{lll}
\forall z \in D \quad \exists w \in B & w \mathrm{R} z
\end{array}\right] \\
& \Longrightarrow \forall z \in D \quad \exists w \in B \quad \exists x \in A \quad x \mathrm{R} w \mathrm{R} z \\
& \Longrightarrow \forall z \in D \quad \exists x \in A \quad x \mathrm{R} z \\
& \Longleftrightarrow A \succsim_{\mathrm{R}} D \text {, }
\end{aligned}
$$

where the second implication assigns $y=w$ and the third uses the transitivity of R. Thus, $\succsim_{\mathrm{R}}$ is transitive. 
Proof of Proposition 2 A. For all $x \in X$ and $\forall A \in \mathfrak{D}_{x}$, we have $A \succsim A$, since $\succsim$ is reflexive, and hence $x \hat{\mathrm{R}} x$. Thus, $\hat{\mathrm{R}}$ is reflexive. B. For all $x, y \in X$, we have

$$
\begin{aligned}
\neg x \hat{\mathrm{R}} y & \Longleftrightarrow \neg \forall A \in \mathfrak{D}_{x} \quad \exists B \in \mathfrak{D}_{y} \quad A \succsim B \\
& \Longleftrightarrow \exists A \in \mathfrak{D}_{x} \quad \forall B \in \mathfrak{D}_{y} \neg A \succsim B \\
& \Longrightarrow \exists A \in \mathfrak{D}_{x} \quad \forall B \in \mathfrak{D}_{y} \quad B \succsim A \\
& \Longrightarrow \forall B \in \mathfrak{D}_{y} \quad \exists A \in \mathfrak{D}_{x} \quad B \succsim A \\
& \Longleftrightarrow y \hat{\mathrm{R}} x,
\end{aligned}
$$

where the third implication uses the completeness of $\succsim$. Thus, $\hat{\mathrm{R}}$ is complete. C. For all $x, y, z \in X$ we have

$$
\begin{aligned}
x \hat{\mathrm{R}} y \hat{\mathrm{R}} z & \Longleftrightarrow\left[\forall A \in \mathfrak{D}_{x} \quad \exists B \in \mathfrak{D}_{y} \quad A \succsim B\right] \wedge\left[\forall D \in \mathfrak{D}_{y} \quad \exists E \in \mathfrak{D}_{z} \quad D \succsim E\right] \\
& \Longrightarrow \forall A \in \mathfrak{D}_{x} \quad \exists B \in \mathfrak{D}_{y} \quad \exists E \in \mathfrak{D}_{z} \quad A \succsim B \succsim E \\
& \Longrightarrow \forall A \in \mathfrak{D}_{x} \quad \exists E \in \mathfrak{D}_{z} \quad A \succsim E \\
& \Longleftrightarrow x \hat{\mathrm{R}} z,
\end{aligned}
$$

where the second implication assigns $D=B$ and the third uses the transitivity of $\succsim$. Thus, $\hat{R}$ is transitive.

Proof of Proposition 3 For all $A, D \in \mathfrak{D}$ and $\mathfrak{B} \subset \mathfrak{D}$ that covers $D$, we have

$$
\begin{aligned}
& \forall B \in \mathfrak{B} A \succ B \Longrightarrow \forall B \in \mathfrak{B} B \succsim A \\
& \Longleftrightarrow \forall B \in \mathfrak{B} \quad B \beth_{\mathrm{R}} A \\
& \Longleftrightarrow \forall B \in \mathfrak{B} \neg \forall y \in A \quad \exists x \in B \quad x \mathrm{R} y \\
& \Longleftrightarrow \forall B \in \mathfrak{B} \exists y \in A \quad \forall x \in B \neg x \mathrm{R} y \\
& \Longrightarrow \exists z \in A \quad \forall B \in \mathfrak{B} \quad \exists y \in A \quad \forall x \in B \quad[z \mathrm{R} y \wedge \neg x \mathrm{R} y] \\
& \Longrightarrow \exists z \in A \quad \forall B \in \mathfrak{B} \forall x \in B \neg x \mathrm{R} z \\
& \Longrightarrow \exists z \in A \quad \forall x \in D \neg x \mathrm{R} z \\
& \Longleftrightarrow \neg \forall z \in A \quad \exists x \in D \quad x \mathrm{R} z \\
& \Longleftrightarrow D \searrow_{\mathrm{R}} A \\
& \Longleftrightarrow D \nsucc A \\
& \Longleftrightarrow[A \succsim D \wedge D \succsim A] \\
& \Longleftrightarrow A \succ D \text {, }
\end{aligned}
$$

where the fifth implication follows from $G(A, \mathrm{R}) \neq \emptyset$, the sixth from the transitivity of $\mathrm{R}$, the seventh from the fact that $\mathfrak{B}$ covers $D$, and the eleventh from the completeness of R. Hence, Cover Dominance holds.

Proof of Proposition 4 A. Suppose that Implicit Optima fails, in which case $\exists A \in \mathfrak{D}$ such that $\forall x \in A$ we can find a $B_{x} \in \mathfrak{D}_{x}$ with $B_{x} \succsim A$. Since $\succsim$ is complete, we have 
$A \succsim B_{x}$ and thus $A \succ B_{x}$. We have also $A \subset \bigcup_{x \in A} B_{x}$, and so Cover Dominance implies that $A \succ A$, a contradiction. B. For all $A, D \in \mathfrak{D}$ and $\mathfrak{B} \subset \mathfrak{D}$ that covers $D$, we have

$$
\begin{aligned}
\forall B \in \mathfrak{B} \quad A \succ B & \Longrightarrow \exists x \in D \quad \exists B_{x} \in \mathfrak{B} \cap \mathfrak{D}_{x} \quad A \succ B_{x} \succsim D \\
& \Longleftrightarrow \exists x \in D \quad \exists B_{x} \in \mathfrak{B} \cap \mathfrak{D}_{x} \quad\left[A \succsim B_{x} \succsim D \wedge B_{x} \succsim A\right] \\
& \Longrightarrow D \succsim A \\
& \Longleftrightarrow[A \succsim D \wedge D \succsim A] \\
& \Longleftrightarrow A \succ D,
\end{aligned}
$$

where the first implication follows from Implicit Optima, the third from the transitivity of $\succsim$, and the fourth from the completeness of $\succsim$. Hence, Cover Dominance holds.

Proof of Proposition 5 We have that

$$
\begin{aligned}
\forall A & \in \mathfrak{D} \exists x \in A \quad \forall B \in \mathfrak{D}_{x} B \succsim A \\
& \Longleftrightarrow \forall A \in \mathfrak{D} \exists x \in A \quad \forall B \in \mathfrak{D}_{x} \quad \forall y \in A \quad \exists E \in \mathfrak{D}_{y} \quad B \succsim E \\
& \Longleftrightarrow \forall A \in \mathfrak{D} \exists x \in A \quad \forall y \in A \quad \forall B \in \mathfrak{D}_{x} \exists E \in \mathfrak{D}_{y} \quad B \succsim E \\
& \Longleftrightarrow \forall A \in \mathfrak{D} \exists x \in A \quad \forall y \in A \quad x \hat{\mathrm{R}} y \\
& \Longleftrightarrow \forall A \in \mathfrak{D} \quad G(A, \hat{\mathrm{R}}) \neq \emptyset,
\end{aligned}
$$

where the initial assertion is Implicit Optima. Hence, $G(\cdot, \hat{\mathrm{R}})$ is nonempty.

Proof of Proposition 6 For all $A, B \in \mathfrak{D}$, we have

$$
\begin{aligned}
A \succsim B & \Longleftrightarrow \exists x \in A \quad \forall D \in \mathfrak{D}_{x} \quad D \succsim A \succsim B \\
& \Longleftrightarrow \exists x \in A \quad \forall D \in \mathfrak{D}_{x} \quad D \succsim B \\
& \Longleftrightarrow \forall y \in B \quad \exists x \in A \quad \forall D \in \mathfrak{D}_{x} \quad D \succsim B \\
& \Longrightarrow \forall y \in B \quad \exists x \in A \quad x \hat{\mathrm{R}} y \\
& \Longleftrightarrow A \succsim_{\hat{\mathrm{R}}} B,
\end{aligned}
$$

where the first implication follows from Implicit Optima and the second from the transitivity of $\succsim$. Hence, we have $\succsim \subset \succsim_{\hat{R}}$.

Proof of Proposition 7 For all $A, D \in \mathfrak{D}$ and $\mathfrak{B} \subset \mathfrak{D}$ that covers $D$, we have

$$
\forall B \in \mathfrak{B} A \succsim B \Longrightarrow \exists x \in D \quad \exists B_{x} \in \mathfrak{B} \cap \mathfrak{D}_{x} A \succsim B_{x} \succsim D \Longrightarrow A \succsim D,
$$

where the first implication follows from Implicit Optima and the second from the transitivity of $\succsim$. Hence, Weak Cover Dominance holds.

Proof of Proposition 8 For all $A, B \in \mathfrak{D}$, we have

$$
A \succsim_{\hat{\mathrm{R}}} B \Longleftrightarrow \forall y \in B \quad \exists x \in A \quad x \hat{\mathrm{R}} y
$$




$$
\begin{aligned}
& \Longleftrightarrow \forall y \in B \quad \exists x \in A \quad \forall D \in \mathfrak{D}_{x} \quad \exists E_{y} \in \mathfrak{D}_{y} \quad D \succsim E_{y} \\
& \Longrightarrow \forall y \in B \quad \exists E_{y} \in \mathfrak{D}_{y} \quad A \succsim E_{y} \\
& \Longrightarrow A \succsim B
\end{aligned}
$$

where the fourth implication follows from Weak Cover Dominance. Hence, we have $\succsim_{\hat{\mathrm{R}}} \subset \succsim$.

Proof of Theorem 1 If $\succsim$ is rationalized by an order $\mathrm{R}$ with $G(\cdot, \mathrm{R})$ nonempty, then Menu Order holds by Corollary 1 and Cover Dominance holds by Proposition 3. Conversely, if Menu Order and Cover Dominance hold, then Implicit Optima holds by Proposition 4A, Weak Cover Dominance holds by Proposition 7, $\hat{\mathrm{R}}$ rationalizes $\succsim$ by Propositions 6 and $8, \hat{\mathrm{R}}$ is an order by Corollary 2 , and $G(\cdot, \hat{\mathrm{R}})$ is nonempty by Proposition 5.

Proof of Proposition 9 Suppose Implicit Optima fails, in which case $\exists A \in \mathfrak{D}$ such that $\forall x \in A$ we can find a $B_{x} \in \mathfrak{D}_{x}$ with $B_{x} \nsucceq A$. Since $\mathfrak{D}$ is finite, the set $\mathfrak{B}=\left\{B_{x}\right.$ : $x \in A\} \subset \mathfrak{D}$ is also finite. Moreover, since $\succsim$ is an order, $\exists y \in A$ such that $\forall x \in A$ we have $B_{y} \succsim B_{x}$. Observing that $A \subset \cup \mathfrak{B}$, Weak Cover Dominance now implies that $B_{y} \succsim A$, contradicting $B_{y} \nsucceq A$.

Proof of Proposition 10 Let $A, B \in \mathfrak{D}$ be such that $A \cup B \in \mathfrak{D}$ and $A \succsim B$. We have $A \succsim A$ since $\succsim$ is reflexive and therefore $A \succsim A \cup B$ by Weak Cover Dominance. Moreover, we have $A \cup B \succsim A \cup B \supset A$ since $\succsim$ is reflexive, and it follows that $A \cup B \succsim A$ by Weak Cover Dominance. Thus, $A \sim A \cup B$, and Kreps Consistency holds.

Proof of Proposition 11 Given $A, B \in \mathfrak{D}$ with $A \subset B$, suppose that $B \nsucceq A$. Then, $A \succsim B$ since $\succsim$ is complete and $A \cup B=B \in \mathfrak{D}$ since $A \subset B$, so that $A \sim A \cup B=B$ by Kreps Consistency. But this contradicts $B \not A$.

Proof of Proposition 12 Given $A, D \in \mathfrak{D}$ and $\mathfrak{B} \subset \mathfrak{D}$ that covers $D$, suppose that $\forall B \in \mathfrak{B}$ we have $A \succsim B$. Since $\mathfrak{D}$ is finite, $\mathfrak{B} \subset \mathfrak{D}$ is finite and can be enumerated as $\mathfrak{B}=\left\{B_{1}, \ldots, B_{n}\right\}$. For each $k \leq n$, write $E_{k}:=\bigcup_{i=1}^{k} B_{i}$ and note that both $E_{k} \in \mathfrak{D}$ and $A \cup E_{k} \in \mathfrak{D}$ since $\mathfrak{D}$ is closed under union. Since $A \succsim B_{1}$, we have $A \sim A \cup B_{1}=A \cup E_{1}$ by Kreps Consistency. [Inductive step begins.] Suppose that for some $k<n$ we have $A \sim A \cup E_{k}$. Since $A \succsim B_{k+1}$ and $\succsim$ is transitive, it follows that $A \cup E_{k} \succsim B_{k+1}$. But then

$$
A \sim A \cup E_{k} \sim\left[A \cup E_{k}\right] \cup B_{k+1}=A \cup E_{k+1},
$$

using Kreps Consistency. [Inductive step ends.] By induction, we can conclude that $A \sim A \cup E_{n}$. Since $D \subset \cup \mathfrak{B}=E_{n} \subset A \cup E_{n}$ and $\succsim$ is complete, we have also $A \cup E_{n} \succsim D$ by Proposition 11 , and so $A \succsim D$ since $\succsim$ is transitive. Hence, Weak Cover Dominance holds.

Proof of Proposition 13 If Weak Cover Dominance holds, then Weak Union Dominance is immediate. Moreover, $\forall A, B \in \mathfrak{D}$ if $A \subset B$ then since $\succsim$ is reflexive we have $B \succsim B$ and thus $B \succsim A$ by Weak Cover Dominance. Hence, Monotonicity holds. 
For the converse, suppose Monotonicity and Weak Union Dominance hold and take any $A, D \in \mathfrak{D}$ and $\mathfrak{B} \subset \mathfrak{D}$ that covers $D$. Since $\mathfrak{D}$ is closed under union, we have $\bigcup \mathfrak{B} \in \mathfrak{D}$. If for each $B \in \mathfrak{B}$ we have $A \succsim B$, then $A \succsim \cup \mathfrak{B}$ by Weak Union Dominance, and since $D \subset \bigcup \mathfrak{B}$ we have $\bigcup \mathfrak{B} \succsim D$ by Monotonicity. But then $A \succsim \bigcup \mathfrak{B} \succsim D$, and so $A \succsim D$ since $\succsim$ is transitive. Hence, Weak Cover Dominance holds.

\section{References}

Ahn, D.S., Sarver, T.: Preference for flexibility and random choice. Econometrica 81, 341-361 (2013)

Barbera, S., Bossert, W., Pattanaik, P.K.: Ranking sets of objects. In: Barbera, S., Hammond, P., Seidl, C. (eds.) Handbook of Utility Theory, Chapter 17, vol. 2, pp. 893-977. Springer, New York (2004)

Bossert, W., Sprumont, Y., Suzumura, K.: Rationalizability of choice functions on general domains without full transitivity. Soc. Choice Welf. 27, 435-458 (2006)

Chambers, C.P., Echenique, F., Shmaya, E.: The axiomatic structure of empirical content. Am. Econ. Rev. 104, 2303-2319 (2014)

Dekel, E., Lipman, B.L., Rustichini, A.: Representing preferences with a unique subjective state space. Econometrica 69, 891-934 (2001)

Dekel, E., Lipman, B.L., Rustichini, A.: Temptation-driven preferences. Rev. Econ. Stud. 76, 937-971 (2009)

Epstein, L.G., Marinacci, M., Seo, K.: Coarse contingencies and ambiguity. Theor. Econ. 2, 355-394 (2007) Ergin, H.: Costly contemplation. Unpublished manuscript (2003)

Gul, F., Pesendorfer, W.: Temptation and self-control. Econometrica 69, 1403-1435 (2001)

Gul, F., Pesendorfer, W.: The simple theory of temptation and self-control. Unpublished manuscript (2005)

Kreps, D.M.: A representation theorem for 'preference for flexibility'. Econometrica 47, 565-577 (1979)

Lipman, B.L., Pesendorfer, W.: Temptation. In: Acemoglu, D., Arellano, M., Dekel, E. (eds.) Advances in Economics and Econometrics: Tenth World Congress, Chapter 8, vol. 1, pp. 243-288. Cambridge University Press, New York (2013)

Nehring, K.: Preference for flexibility in a Savage framework. Econometrica 67, 101-119 (1999)

Olszewski, W.: Preferences over sets of lotteries. Rev. Econ. Stud. 74, 567-595 (2007)

Richter, M.K.: Revealed preference theory. Econometrica 34, 635-645 (1966)

Richter, M.K.: Rational choice. In: Chipman, J.S., Hurwicz, L., Richter, M.K., Sonnenschein, H.F. (eds.) Preferences, Utility, and Demand, Chapter 2, pp. 29-58. Harcourt Brace Jovanovic, New York (1971)

Sen, A.K.: Quasi-transitivity, rational choice, and collective decisions. Rev. Econ. Stud. 36, 381-393 (1969)

Sen, A.K.: Choice functions and revealed preference. Rev. Econ. Stud. 38, 307-317 (1971)

Stovall, J.E.: Multiple temptations. Econometrica 78, 349-376 (2010)

Tyson, C.J.: Behavioral implications of shortlisting procedures. Soc. Choice Welf. 41, 941-963 (2013) 